\title{
Turbulence and Singularity of the Velocity Field
}

\author{
By \\ Shigeo KIDA*
}

\begin{abstract}
The small-scale properties of turbulence are related to the singularity of the velocity field in the inviscid limit. The energy spectrum in the inertial range is expressed in terms of the fractal dimension and Hölder's exponent of the singularity. It is pointed out that the entrainment process is important in the dynamics of active eddies in the $\beta$-model theory of turbulence.
\end{abstract}

\section{§1. Introduction}

The motion of an incompressible viscous fluid, including turbulence, is governed by the Navier-Stokes equation and the continuity equation,

$$
\begin{gathered}
\frac{\partial \boldsymbol{u}}{\partial t}+(\boldsymbol{u} \cdot \boldsymbol{\nabla}) \boldsymbol{u}=-\frac{1}{\rho} \nabla p+\frac{1}{R} \nabla^{2} u, \\
\boldsymbol{\nabla} \cdot \boldsymbol{u}=0,
\end{gathered}
$$

where $\boldsymbol{u}(\boldsymbol{x}, t)$ is the velocity, $\boldsymbol{x}$ the space coordinate, $t$ the time, $\rho$ the (constant) density, $p$ the pressure, and $R$ the Reynolds number which is defined by the representative length and velocity and the kinematic viscosity of fluid.

Turbulence is usually realized at extremely large values of $R$. We are therefore interested in the asymptotic behaviour of the velocity field in the limit of large $R$ (inviscid limit). Since $1 / R$, the coefficient of the highest derivative term with respect to $\boldsymbol{x}$ in (1.1), tends to zero in this limit, the solution to (1.1) and (1.2) may become singular in space. The velocity gradient then takes extremely large values in some narrow regions in space, which will be called the singular regions, and takes ordinary values elsewhere. Such a singular nature of the velocity field brings about the non-zero viscous dissipation of energy or enstrophy in the inviscid limit. There is a conjecture that the small-scale properties of turbulence, e.g. the energy spectrum function in the inertial range may

Received February 7, 1983.

* Research Institute for Mathematical Sciences, Kyoto University, Kyoto 606, Japan. 
be related to the singularity [1].

In this article, we will consider homogeneous isotropic turbulence, paying special attention to its connection with the singularity of the velocity field in the inviscid limit. In Section 2, the non-zero effect of viscosity in the inviscid limit will be emphasized through the decay laws of energy and enstrophy. It will be argued that the occurrence of the singularity is different between two- and three-dimensional cases. In Section 3, we will consider the energy-cascade model, including the effect of intermittency of turbulence. These models express in a clear-cut way the small-scale structure of turbulence. It will be pointed out that the entrainment process is important in the dynamics of small-scale eddies in the $\beta$-model theory. In Section 4 , we will try to characterize the singularity by two parameters which represent the fractal dimension and the strength of the singularity and to relate them with the energy spectrum in the inertial range. Section 5 is devoted to concluding remarks.

\section{§ 2. Inviscid Energy and Enstrophy Dissipation}

In the inviscid limit the coefficient of the viscous term in (1.1) tends to zero but this term cannot be neglected completely because of the appearance of a singularity in the velocity field. Rather it plays a significant role in the energy and enstrophy dissipation in three- and two-dimensional cases respectively.

Multiplying (1.1) by $\boldsymbol{u}(\boldsymbol{x}, t)$ and taking an ensemble average, we get

$$
\frac{d}{d t} \mathscr{E}(t)=-\frac{2}{R} Q(t) \quad(\equiv-\varepsilon(t), \text { say })
$$

where

$$
\mathscr{E}(t) \equiv \frac{1}{2}\left\langle|\boldsymbol{u}(\boldsymbol{x}, t)|^{2}\right\rangle=\int_{0}^{\infty} E(k, t) d k
$$

is the kinetic energy of fluid motion per unit mass, $E(k, t)$ is the energy spectrum function, $k$ being the wavenumber,

$$
Q(t) \equiv \frac{1}{2}\left\langle|\omega(x, t)|^{2}\right\rangle=\int_{0}^{\infty} k^{2} E(k, t) d k
$$

is the enstrophy,

$$
\boldsymbol{\omega}=\boldsymbol{\nabla} \times \boldsymbol{u}
$$

is the vorticity and $\varepsilon(t)$ is the energy-dissipation rate due to viscosity. \langle\rangle de- 
notes an ensemble average. The velocity field has been assumed to be statistically homogeneous and isotropic.

The curl of (1.1) gives the vorticity equation

$$
\frac{\partial \boldsymbol{\omega}}{\partial t}+(\boldsymbol{u} \cdot \boldsymbol{\nabla}) \boldsymbol{\omega}=(\boldsymbol{\omega} \cdot \boldsymbol{\nabla}) \boldsymbol{u}+\frac{1}{R} \nabla^{2} \boldsymbol{\omega} .
$$

On taking an ensemble average of (2.5) multiplied by $\boldsymbol{\omega}(\boldsymbol{x}, t)$, we get

$$
\frac{d}{d t} Q(t)=\langle\omega \cdot[(\omega \cdot \nabla) u]\rangle-\frac{2}{R} P(t) \quad(\equiv-\eta(t), \text { say }),
$$

where

$$
P(t) \equiv \frac{1}{2}\left\langle|\boldsymbol{\nabla} \times \boldsymbol{\omega}|^{2}\right\rangle
$$

is the palinstrophy and $\eta(t)$ is the enstrophy dissipation rate.

2.1. Three-dimensional turbulence. The first term on the r.h.s. of (2.6) is proportional to the mean gradient of velocity along the direction of vorticity. This term is known experimentally to be positive definite and is understood to represent the increase of enstrophy due to stretching of the vortex filaments.

It is generally accepted by many analytical theories of turbulence [2-4] that the energy dissipation rate remains finite in the inviscid limit in three-dimensional turbulence,

$$
\varepsilon>0 \text { as } R \rightarrow \infty
$$

Incidentally, as will be discussed in the next section, Kolmogorov [5] derived the famous $-5 / 3$ law for the energy spectrum (3.1) under the hypohtesis (2.8). Combination of (2.1) and (2.8) leads to

$$
Q \rightarrow \infty \text { as } R \rightarrow \infty,
$$

i.e. enstrophy is a quantity which diverges in the inviscid limit.

Figure 1 shows the change of turbulent energy in time, which is the result of the modified zero fourth order cumulant approximation [6]. Cases I and II refer to different initial conditions. For such large Reynolds numbers, the energy is nearly constant up to a critical time but after that it abruptly decays according to a power law $\mathscr{E} \propto t^{-s}$. The critical time and the power $s$ are different for different initial conditions but they tend to some finite values in the inviscid limit. Of course, the enstrophy diverges to infinity in the inviscid limit after the critical time. 


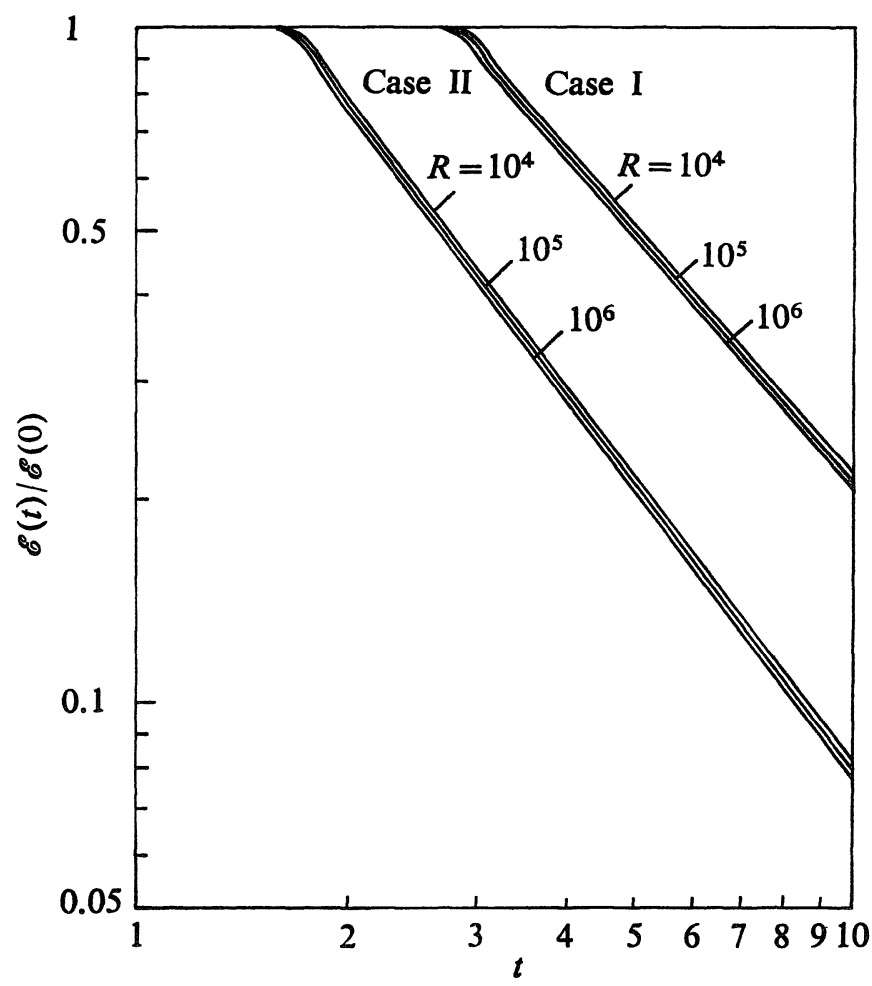

Fig. 1. Decay of energy in three-dimensional turbulence (after Tatsumi and Kida [6]).

In this context, it has been shown numerically that enstrophy does blow up at a finite time for a velocity field which obeys the inviscid (Euler) equation when it starts with a regular initial condition called Taylor Green's vortex [7]. This is consistent with the above result concerning a finite critical time.

2.2. Two-dimensional turbulence. In the case of two-dimensional flow, the first term on r.h.s. of (2.6) is identically zero, i.e. there is no mechanism of stretching of vortex filaments. The change of enstrophy in time is then written as

$$
\frac{d}{d t} Q(t)=-\frac{2}{R} P(t)=-\eta(t) \quad(<0)
$$

This shows that enstrophy, which is positive definite, decreases in time and never diverges to infinity in contrast with the three-dimensional case.

In view of (2.1) we can see that

$$
\varepsilon \rightarrow 0 \text { as } R \rightarrow \infty
$$


i.e. there is no energy dissipation in the inviscid limit.

Now let $\boldsymbol{\chi}(\boldsymbol{x}, t)$ be the curl of the vorticity $\boldsymbol{\omega}=\omega \hat{z, z}$ being a unit vector normal to the plane of the two-dimensional motion of fluid,

$$
\boldsymbol{\chi}=\boldsymbol{\nabla} \times(\omega \hat{z}) .
$$

The curl of (2.5) gives

$$
\frac{\partial \boldsymbol{\chi}}{\partial t}+(\boldsymbol{u} \cdot \boldsymbol{\nabla}) \boldsymbol{\chi}=(\boldsymbol{\chi} \cdot \boldsymbol{\nabla}) \boldsymbol{u}+\frac{1}{R} \nabla^{2} \boldsymbol{\chi}
$$

The change of palinstrophy in time is then written as

$$
\frac{d}{d t} P(t)=\langle\chi \cdot[(\chi \cdot \nabla) u]\rangle-\frac{1}{R}\left\langle|\nabla \times \chi|^{2}\right\rangle .
$$

The first term on r.h.s. of (2.14) is proportional to the mean gradient of the velocity along the equi-vorticity lines. If it is positive, this term contributes to the increase of the vorticity gradient or palinstrophy [8]. In two-dimensional turbulence therefore it is possible that palinstrophy goes to infinity and viscous dissipation of enstrophy [see (2.10)] remains finite in the inviscid limit,

$$
\eta>0 \quad \text { as } \quad R \rightarrow \infty \text {. }
$$

On the other hand, it has been proved already by Kato [9] that a velocity field which obeys the two-dimensional Euler equation remains regular at any finite time if it is so initially. Therefore, even if palinstrophy diverges, the critical time at which the divergence takes place must go to infinity with $R$.

Figure 2 shows the decay of enstrophy for various values of $R$, which is due to the modified zero fourth order cumulant approximation [10]. (I) and (II) represent different initial conditions. As seen in this figure or as derived exactly by examining the asymptotic behaviour in the inviscid limit of solution to the equation for energy spectrum function [11], there exists a critical time

$$
t_{c}=O\left[(\ln R)^{1 / 2}\right]
$$

before which the enstrophy is nearly constant and after which it decays according to

$$
Q(t)=4 t^{-1} \ln (R t) .
$$

Note that the critical time $t_{c}$ goes to infinity with $R$ as expected.

The above results are based upon an approximate theory of turbulence and the order of the critical time (2.16) has not been proved exactly. However, it 


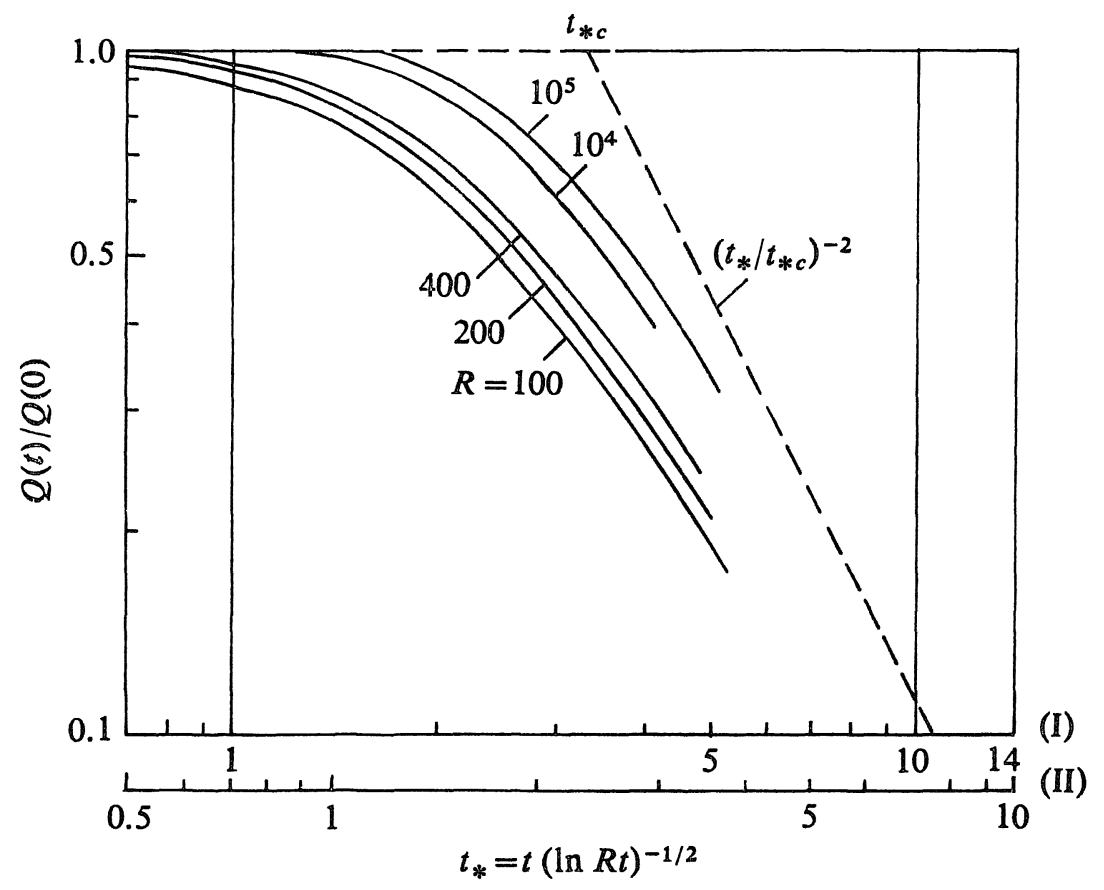

Fig. 2. Decay of enstrophy in two-dimensinal turbulence (after Tatsumi and Yanase [10]).

is at least probable that there exists such a critical time that is finite for finite $R$ and increases to infinity with $R$.

\section{§3. Energy Cascade Model}

3.1. Kolmogorov's $\mathbf{1 9 4 1}$ theory. The turbulent velocity field may be regarded as an assembly of a number of eddies of various sizes and shapes, which are moving about in a very complicated manner.

The following cascade process is generally accepted as a mechanism of energy-transfer in three-dimensional turbulence. The above eddies are stretched at random and deformed into highly complicated thinner eddies through the nonlinear interaction in the Navier-Stokes equation. These stretched eddies disintegrate into a number of smaller ones owing to their instability. This process of disintegration continues down to the smallest eddies which dissipate into heat by the action of viscosity. As a result there exists a (continuous) hierarchy of eddies. 
The energy spectrum at small wavenumbers, which reflects the motion of large-scale eddies, is different for different kinds of turbulence since the large eddies are excited directly by external forces. At large wavenumbers (smallscale eddies), on the other hand, the turbulence is thought to be in a somewhat universal state, since the small-scale eddies are created through a number of steps of the cascade process. The characteristic times of the constituent eddies decreases with their size, or the energy spectrum at larger wavenumbers has smaller characteristic time. Therefore, even if the turbulence is non-steady as a whole, the components of large wavenumbers at least could be in a quasisteady and quasi-equilibrium state.

Kolmogorov [5] assumed that such an equilibrium state of large wavenumber components is statistically homogeneous, isotropic and steady, and that it depends upon only two parameters, the energy dissipation rate $\varepsilon$ and the viscosity of fluid $v$. Moreover, when the Reynolds number is very large, there appears a range of wavenumbers which is not affected by viscosity at the lowest part of the above equilibrium range. The energy spectrum there depends only upon $\varepsilon$. In this inertial range $\varepsilon$ is equal to the rate of energy-transfer in the cascade process since energy is transferred locally in wavenumber space and the energy loss due to viscosity is negligible. A dimensional argument then leads to

$$
E(k) \propto \varepsilon^{2 / 3} k^{-5 / 3} .
$$

This $-5 / 3$ law of the energy spectrum is observed in various kinds of large Reynolds number turbulence $[12,13]$.

3.2. Intermittency. According to Kolmogorov's 1941 theory, the motion of small-scale components of turbulence is statistically independent of that of largescale components. The probability distribution of the velocity gradient, for example, should be a universal function which is independent of the Reynolds number, since they are determined mainly by the motion of small-scale components.

There is, however, a lot of experimental evidence in conflict with the above picture [14-17]. The kurtosis of the probability distribution of the velocity gradient was found to increase with the Reynolds number, i.e. the statistics of the small-scale components obviously depend on that of large-scale components. The large values of kurtosis mean that the probability distribution of the velocity gradient has a high peak near the origin and long tails far away from the origin. 
This can be understood most reasonably by supposing that small-scale components are excited not uniformly in space but rather intermittently. The fact that the kurtosis increases with $R$ implies that the velocity field is more intermittent in smaller scales, since the smaller-scale motion is excited for larger Reynolds numbers.

In view of such an intermittent structure of turbulence, various attempts have been made to modify Kolmogorov's 1941 theory so as to incorporate such effects. Among others, the log-normal and $\beta$-model theories are remarkable.*)

Kolmogorov [19] and Obukhof [20] estimated the correction due to the intermittency by assuming that the rate of viscous dissipation is distributed in space according to a log-normal distribution. In the original theory the averaged dissipation rate was used as a governing parameter.

A more simple and intuitive description of the energy cascade process including the intermittency effect is the $\beta$-model [21-23], which will be discussed in the next subsection. The idea is that the energy cascade from larger to smaller eddies takes place only in a minor fraction of the whole space.

These two theories give rather small corrections to the energy spectrum function to be detected experimentally. But the correction becomes considerable for the moments of velocity derivatives. The discrepancy between their results also increases with the order of derivative. At the moment, however, it is difficult to say which theory is more consistent with the experimental results partly because of insufficient accuracy of measurement.

In the following we will examine the dynamics of the cascade process in the $\beta$-model theory [24] in the hope that this simple model may give an understanding of the singular structure of the velocity field in the inviscid limit.

3.3. The $\beta$-model. As a simple model of the hierarchical structure of eddies in turbulence, we consider an assembly of a discrete sequence of eddies with length-scales,

$$
l_{n}=2^{-n} l_{0}, \quad n=1,2, \ldots, n_{v},
$$

where $l_{0}$ is the length-scale of the largest eddies roughly corresponding to the energy-containing eddies and $l_{n_{v}}$ is that of the smallest eddies which dissipate into heat by the action of viscosity. The fluid motion is assumed to be active in

*) Mandelbrot [18] has proposed a general model which includes these two models as special cases. 
these eddies and inactive elsewhere. The eddies of size $l_{n}$ will be called $n$-eddies. There are various $n$-eddies of different ages, i.e. from the youngest ones which have just been born from $(n-1)$-eddies, to the oldest which are about to break down into $(n+1)$-eddies.

Let $v_{n}$ be the order of magnitude of the velocity fluctuations in $n$-eddies, and let $\beta_{n}$ be the ratio of the volume occupied by $n$-eddies to that occupied by 0 -eddies. If we assume for simplicity that the 0 -eddies occupy the whole space, the turbulent kinetic energy of $n$-eddies per unit mass is written as

$$
E_{n}=\frac{1}{2} \beta_{n} v_{n}^{2}
$$

The cascade process of energy-transfer in the inertial range is modelled as follows. An $n$-eddy disintegrates into $2^{s}\left[=\left(l_{n} / l_{n+1}\right)^{s}\right](n+1)$-eddies on average during the lifetime $t_{n}$ which is given by the turnover time of $n$-eddies,

$$
t_{n}=l_{n} / v_{n}
$$

The exponent $s$ is called the offspring exponent.

Note that the volume of $n$-eddies increases or decreases in time according as $s$ is greater or less than the space dimension 3. Each eddy does not need to preserve its volume even if the fluid is incompressible and the viscous effect is negligible, because we are describing the velocity field in a coarse-grained sense. In fact, as will be shown later, this volume increases in time.

The mean rate of energy-transfer from $n$-eddies to $(n+1)$-eddies is expressed as

$$
\varepsilon_{n}=E_{n} / t_{n}
$$

Since the effect of viscosity can be neglected in the inertial range, we have the following equation of energy conservation,

$$
\frac{d}{d t} E_{n}=\varepsilon_{n-1}-\varepsilon_{n}
$$

If we denote the number of $n$-eddies per unit mass by $N_{n}$, the rate of change in time of this number is written as

$$
\frac{d}{d t} N_{n}=\left[\frac{l_{n-1}}{l_{n}}\right]^{s} \frac{N_{n-1}}{t_{n-1}}-\frac{N_{n}}{t_{n}} .
$$

It follows from the definitions of $\beta_{n}$ and $N_{n}$ that

$$
\beta_{n}=\frac{N_{n}}{N_{0}}\left[\frac{l_{n}}{l_{0}}\right]^{3}
$$


As stated in Section 3.1, even if the large-scale motion of turbulence is nonsteady, the small-scale motion may be quasi-steady in a statistical sense. This is the case if the characteristic time $t_{n}$ decreases with $n$, as can be checked by the result [see (3.11) and (3.16)]. The steady solution $\left(d E_{n} / d t=d N_{n} / d t=0\right)$ of (3.3)-(3.8) is easily obtained as follows:

$$
\begin{aligned}
& E_{n}=2^{-1 / 3} \varepsilon^{2 / 3} l_{n}^{2 / 3}\left(l_{n} / l_{0}\right)^{(3-D) / 3}, \\
& v_{n}=2^{1 / 3} \varepsilon^{1 / 3} l_{n}^{1 / 3}\left(l_{n} / l_{0}\right)^{-(3-D) / 3}, \\
& t_{n}=2^{-1 / 3} \varepsilon^{-1 / 3} l_{n}^{2 / 3}\left(l_{n} / l_{0}\right)^{(3-D) / 3}, \\
& N_{n}=N_{0}\left(l_{n} / l_{0}\right)^{-D}, \\
& \beta_{n}=\left(l_{n} / l_{0}\right)^{3-D},
\end{aligned}
$$

where $\varepsilon$ is the rate of energy transfer, which is independent of $n$, and $D$ is Mandelbrot's fractal dimension [22], which is related to the offspring exponent $s$ by

$$
D=(3 s-5) / 2 \text {. }
$$

The energy spectrum function of the turbulent velocity fluctuation is derived from (3.9) through the Fourier transformation as

$$
E(k) \approx \varepsilon^{2 / 3} k^{-5 / 3}\left(k l_{0}\right)^{-(3-D) / 3} .
$$

At present, the value of $D$ has not yet been determined theoretically [24], but the available experimental values are included in the range [25]

$$
2.5 \lesssim D \lesssim 2.7 \text {. }
$$

It follows from (3.14) and (3.16) that

$$
3.3 \lesssim s \lesssim 3.5 \text {. }
$$

Kolmogorov's 1941 theory corresponds to the non-intermittent case $(D=3)$, for which (3.14) gives

$$
s=11 / 3(>3) \text {. }
$$

In the present model, an $n$-eddy changes in volume by $\left(l_{n} / l_{n-1}\right)^{s-3}$ times during its lifetime $t_{n}$. Since $s>3$ [see (3.17)], the volume of each eddy actually increases in time, which suggests that the surrounding non-turbulent fluid is steadily entraining into the eddies. Thus, the entrainment process is important in the dynamics of small-scale eddies in fully developed turbulence just as in 
large-scales such as the turbulent mixing-layer*) and the turbulent vortex ring**).

It may be unnecessary to say that although the volume of each eddy increases steadily in time, the fraction of volume occupied by eddies decreases with their scales because smaller eddies have shorter lifetimes [see (3.11) and (3.16)]. This describes the intermittent structure of turbulence.

\section{§4. Singularity of the Velocity Field}

As stated in the introduction, singular regions, which are localized in space, are built up in the velocity field in the limit of large Reynolds number. The existence of such localized singular regions may explain the intermittent structure which was discussed in the preceding section.

Mathematically, little is known about the singularity of the inviscid equation. The analysis of non-viscous cases seems to be much more difficult than that of viscous cases [28].

As a first step to the study of the singularity we will introduce two parameters, the fractal dimension and Hölder's exponent of the singularity and express the power law in the energy spectrum function in terms of these parameters [29].

4.1. Characterization of singularity. The fractal dimension of the singular region is defined as follows. Let us divide the whole of $d$-dimensional space into infinite numbers of boxes of side length $l_{0}$ and select those boxes which contain part of the singular region. Next, subdivide each of the selected boxes into $2^{d}$ smaller boxes of side length $l_{1}=2^{-1} l_{0}$ and select those new boxes which contain part of the singular region. Let $N_{1}$ be defined as the average number of new selected boxes per old one. We repeat this procedure of subdivision $n_{v}$ times until the effect of viscosity becomes important in those boxes of side length $l_{n_{v}}$. We have then a series of numbers $N_{1}, N_{2}, \ldots, N_{n_{v}}$.

Assuming that the singular region has a similar structure in the sense that the ratio $N_{n+1} / N_{n}$ does not depend on $n$, we put

$$
N_{n+1} / N_{n} \equiv 2^{D} .
$$

The exponent $D$ is the fractal dimension of the singular region [30]. Although (4.1) has the same form as (3.12), the definitions of $D$ are slightly different.

*) See for example [26].

**) See for example [27]. 
Remember that the eddies of different ranks may overlap with each other in the $\beta$-model.

The second parameter which characterizes the singular region represents the strength of the singularity. Let the velocity field have the singularity of Hölder's exponent $\sigma$, i.e. the velocity difference $\Delta u$ between two points separated by $\Delta \boldsymbol{x}$ is expressed by

$$
|\Delta u| \propto|\Delta x|^{\sigma} .
$$

If we denote the order of magnitude of velocity fluctuation in the boxes of sidelength $l_{n}$ by $u_{n}$, we have

$$
u_{n} \propto l_{n}^{\sigma} .
$$

For example, a continuous velocity field whose space derivative is discontinuous, a shock-like discontinuity and a velocity field around a vortex filament correspond to $\sigma=1,0$ and -1 respectively.

The average of the square of the velocity difference between two points separated by $\boldsymbol{r}$

$$
B(r) \equiv\left\langle|\boldsymbol{u}(\boldsymbol{x}+\boldsymbol{r})-\boldsymbol{u}(\boldsymbol{x})|^{2}\right\rangle, \quad r=|\boldsymbol{r}|
$$

is called the structure function. We can show this is expressed as

$$
B(r) \propto r^{2 \sigma+d-D}
$$

in the inertial range. The corresponding energy spectrum function is

$$
E(k) \propto k^{-2 \sigma-d+D-1} .
$$

In principle, the parameters $D$ and $\sigma$ should be determined simultaneously by examining the asymptotic behaviour of the solution to the Navier-Stokes equation in the inviscid limit. Unfortunately, this has not yet been done. Here we impose a relation between these parameters and show that several models of turbulence can be understood in a unified manner.

4.2. The three-dimensional case. If the energy-dissipation rate remains finite in the inviscid limit [see (2.8)], it is easy to show that

$$
\sigma=(D-2) / 3 \text {. }
$$

Substituting (4.7) into (4.6), we obtain,

$$
E(k) \propto k^{-(5 / 3)-(3-D) / 3},
$$

which is identical to the $\beta$-model. 
Note that although we have reached the same conclusion as the $\beta$-model, the underlying assumptions are different. The $\beta$-model assumes a finite energy flux in the energy cascade process, while the present theory assumes the finite energy dissipation in the inviscid limit and does not assume the energy cascade process or the localness of the energy transfer at all.

4.3. The two-dimensional case. As stated in Section 2.2, in the two-dimensional case the energy dissipation rate vanishes in the inviscid limit. Instead, it is possible that the enstrophy dissipation rate $\eta$ may remain finite in this limit. If this is so, we obtain, by a similar argument as in the preceding subsection,

$$
\sigma=(D+1) / 3
$$

and

$$
E(k) \propto k^{-3-(2-D) / 3} .
$$

If the velocity field is non-intermittent $(D=2)$, then (4.10) becomes

$$
E(k) \propto k^{-3} \text {. }
$$

This is identical to the spectrum which has been derived on the basis of an enstrophy cascade process [8], [31], [32].

Another model of two-dimensional turbulence was proposed by Saffman [33]. In view of the conservation of vorticity in the inviscid limit, he supposed that the velocity field is composed of a number of vortex regions of uniform vorticity which are separated by very thin boundary layers, and derived

$$
E(k) \propto k^{-4} \text {. }
$$

This velocity field corresponds to the case $D=1$ and $\sigma=1$, since the singular regions (boundary layers) are one-dimensional lines and the derivative of the velocity across the singular lines is discontinuous. The general expression of the energy spectrum (4.6) then leads to Saffman's spectrum (4.12).

Incidentally, this spectrum corresponds to the state in which the enstrophy dissipation rate vanishes in the inviscid limit,

$$
\eta \rightarrow 0 \text { as } R \rightarrow \infty .
$$

Spectra (4.10) and (4.12) therefore correspond to the states of finite and zero inviscid enstrophy dissipation respectively. Which is actually realized in two-dimensional turbulence? In spite of extensive numerical simulations, however, no result has yet been obtained which discriminates these two spectra 
clearly.

4.4. Other models of turbulence. The simplest singularities in the inviscid Navier-Stokes equation are vortex filaments and sheets.

If the singular region is supposed to be composed of vortex filaments, we have $d-D=2$ irrespective of the dimensionality of turbulence $d$. For such flows the velocity changes in inverse proportion to the distance from the centre of the filament and we have $\sigma=-1$. Then (4.6) yields the energy spectrum

$$
E(k) \propto k^{-1} \text {. }
$$

If, on the other hand, the singular region is made of vortex sheets, we have $d-D=1$ again irrespective of the value of $d$. Since the velocity jumps across the vortex sheet, we have $\sigma=0$. Then it follows from (4.6) that

$$
E(k) \propto k^{-2} \text {. }
$$

These spectra (4.14) and (4.15) are only kinematically possible but are not guaranteed to be realized dynamically. It may be interesting, however, to note that the exponent of the modified Kolmogorov's spectrum (4.8) appears to be in the range between -1 and -2 .

\section{§5. Concluding Remarks}

A turbulent velocity field may be regarded as a non-linear dissipative dynamical system with infinite degrees of freedom which is highly unstable to small disturbances. The study of turbulence therefore could contribute to the development of non-equilibrium statistical mechanics, which has not been established yet so much as equilibrium statistical mechanics.

We have seen in this article that the singularity of the velocity field in the inviscid limit is closely related to the small-scale structure of turbulence and that the energy spectrum function in the inertial range can be expressed in terms of the fractal dimension and Hölder's exponent of the singular region in the velocity field. Mathematically, however, little is known about the analytical properties of solutions to the Navier-Stokes equation in the inviscid limit. It is hoped that more extensive research for the singularity will be performed mathematically, numerically and experimentally. Is it possible to describe turbulence as an assembly of singular regions and to study their statistical properties by applying the notions and techniques of statistical mechanics? 


\section{Acknowledgement}

The author would like to express his cordial thanks to Professor H. K. Moffatt who kindly read the manuscript and made helpful suggestions for improvement.

\section{References}

[1] Mandelbrot, B. B., Fractal: Form, Chance and Dimension, Freeman, San Francisco, 1977.

[2] Brissaud, A., Frisch, U., Léorat, J., Lesieur, M., Mazure, A., Pouquet, A., Sadourny, R. and Sulem, P. L., Annales de Géophysique, 29 (1973) 539.

[3] Orszag, S. A., Lectures on the statistical theory of turbulence. In Fluid Dynamics: Les Houches 1973, eds. R. Balian and J. L. Peube, Gordon and Breach, 1977.

[4] Tatsumi, T., Kida, S. and Mizushima, J., J. Fluid Mech., 85, (1978) 97.

[5] Kolmogorov, A. N., C. R. Acad, Sci. URSS, 30 (1941) 301.

[6] Tatsumi, T. and Kida, S., J. Phys. Soc. Japan, 49 (1980) 2014.

[7] Morf, R. H., Orszag, S. A. and Frisch, U., Phys. Rev. Lett., 44 (1980) 572.

[8] Batchelor, G. K., Phys. Fluids Suppl., 12 (1969) II 233.

[9] Kato, T., Arch. Rat. Mech. Anal., 25 (1967) 188.

[10] Tatsumi, T. and Yanase, S., J. Fluid Mech., 110 (1981) 475.

[11] Kida, S., Phys. Fluids, 24 (1981) 604.

[12] Grant, H. L., Stewart, R. W. and Moilliet, A., J. Fluid Mech., 12 (1962) 241.

[13] Champagne, F. H., J. Fluid Mech., 86 (1978) 67.

[14] Batchelor, G. K. and Townsend, A. A., Proc. Roy. Sec., A199 (1949) 238.

[15] Wyngaard, J. C. and Pao, Y. H., Lecture Notes in Physics, 12, Springer-Verlag, (1972) 384.

[16] Frenkiel, F. N., Klebanoff, P. S. and Huang, T. T., Phys. Fluids, 22 (1979) 1606.

[17] Van Atta, C. W. and Antonia, R. A., Phys. Fluids, 23 (1980) 252.

[18] Mandelbrot, B. B., J. Fluid Mech., 62 (1974) 331.

[19] Kolmogorov, A. N., J. Fluid Mech., 13 (1962) 82.

[20] Obukhov, A. M., J. Fluid Mech., 13 (1962) 77.

[21] Novikhov, E. A. and Stewart, R. W., Isv. Akad. Nauk USSR, Ser. Geophys., 3 (1964) 408.

[22] Mandelbrot, B. B., Lecture Notes in Mathematics, 565 (1976) 121.

[23] Frisch, U., Sulem, P. L. and Nelkin, M., J. Fluid Mech., 87 (1978) 719.

[24] Kida, S., Prog. Theor. Phys., 67 (1982) 1630.

[25] Monin, A. S. and Yaglom, A. M., Statistical Fluid Mechanics: Mechanics of Turbulence, MIT Press, Vol. 2, 1975.

[26] Winant, C. D. and Browand, F. K., J. Fluid Mech., 63 (1974) 237.

[27] Maxworthy, T., J. Fluid Mech., 51 (1972), 15.

[28] Scheffer, V., Commun. Math. Phys., 73 (1980) 1.

[29] Kida, S. and Tatsumi, T., submitted to J. Phys. Soc. Japan.

[30] Mandelbrot, B. B., Lecture Notes in Mathematics, 615 (Springer-Verlag, 1977) 83. 
[31] Kraichnan, R. H., Phys. Fluids, 10 (1967) 1417.

[32] Leith, C. E., Phys. Fluids, 11 (1968) 671.

[33] Saffman, P. G., Stud. Appl. Math., 50 (1971) 377. 